\title{
Retinoscopia com luz em faixa em cães fácicos, afácicos e pseudofácicos
}

\author{
[Streak retinoscopy in phakic, aphakic, and pseudophakic dogs] \\ L.A.L. Mobricci ${ }^{1}$, J.J.T. Ranzani ${ }^{1}$, P.V.M. Steagall ${ }^{1}$, A.C.L. Rodrigues ${ }^{2}$, \\ L.R. Carvalho ${ }^{3}$, C.V.S. Brandão ${ }^{1}$ \\ ${ }^{1}$ Faculdade de Medicina Veterinária e Zootecnia - UNESP - Botucatu, SP \\ ${ }^{2}$ Faculdade de Medicina - UNESP - Botucatu, SP \\ ${ }^{3}$ Instituto de Biociências - UNESP - Botucatu, SP
}

\begin{abstract}
RESUMO
Avaliou-se o erro refracional em 40 cães submetidos ou não à facectomia por facoemulsificação, por meio de retinoscopia com luz em faixa. Distribuídos em quatro grupos de 10 animais cada, foram designados por cães fácicos, cães afácicos (CA), cães pseudofácicos com implante de duas lentes intra-oculares em piggyback (PP) e cães pseudofácicos com implante de uma lente intra-ocular veterinária de 41D (PL). As avaliações foram realizadas por ceratometria, ecobiometria ultra-sônica e retinoscopia. A média da curvatura corneana foi de $40,46 \pm 2,44 \mathrm{D}$. O poder dióptrico obtido por retinoscopia foi de $18,50 \mathrm{D}$ no grupo CA, 5,25D no grupo PP e 2,00D no grupo PL. A retinoscopia com luz em faixa constituiu um método eficaz para avaliar a refração ocular em cães, mas foi dificultada quando da presença de opacidade de meios oculares. Todos os animais apresentaram-se hipermétropes, em diferentes graus; o astigmatismo ceratométrico cirurgicamente induzido foi discreto.
\end{abstract}

Palavras-chave: cão, retinoscopia, fácicos, afácicos, pseudofácicos

\begin{abstract}
The refraction error in dogs submitted or not to phakectmy, by phakoemulsification by streak retinoscopy was evaluated. Forty dogs were distributed in four groups of 10 animals each, and were designated by phakic, aphakic (AD), pseudo-phakic with two piggyback intraocular lenses (PD), and pseudo-phakic with one intraocular $41 D$ veterinary lens implant (LD). The evaluation was performed by keratometry, ultrasonic echobiometry, and retinoscopy. The mean corneal curvature was $40.46 \pm 2.44 D$. The dioptric power obtained by retinoscopy was $18.50 D$ in AD group, 5.25D in PD group, and 2.00D in LD group. The retinoscopy was an efficient method to evaluate ocular refraction in dogs, but was more complex in ocular opacity presence. All animals presented different levels of hypermetropy, and the surgically induced astigmatism was discrete.
\end{abstract}

Keywords: dog, retinoscopy, phakia, aphakia, pseudo-phakia

\section{INTRODUÇÃO}

Os métodos de avaliação do estado óptico do olho e os meios empregados para a sua correção desempenham importante papel no tratamento de muitas condições oftálmicas (Duke-Elder, 1997). As técnicas de ceratometria, retinoscopia e de ecobiometria ocular permitem intercambiar informações complementares. A combinação

Recebido em 11 de março de 2008

Aceito em 23 de outubro de 2008

Endereço para correspondência (corresponding address)

R. Arthur Carlos Ferreira, 63 - 12244-451 - São José dos Campos, SP

E-mail: lumobricci@yahoo.com.br desses dados propicia a melhora na seleção e avaliação de pacientes a serem submetidos à facectomia e auxilia na identificação de problemas de desempenho (Murphy et al., 1997; Gelatt, 1999). A avaliação da refração ocular é recomendada nos exames de triagem para seleção de animais em funções específicas (Murphy et al., 1997), assim como para a correção de ametropias em animais fácicos, afácicos e pseudofácicos (Davidson et al., 1993). 
A ametropia é o estado de refração ocular, no qual os raios de luz paralelos de um objeto distante não se focalizam na retina, estando o olho em repouso. $\mathrm{Na}$ ametropia axial, o olho pode ter comprimento anteroposterior mais longo, causando miopia, ou mais curto, proporcionando hipermetropia. $\mathrm{Na}$ ametropia refrativa, o poder dióptrico é excessivo na miopia e insuficiente na hipermetropia (Duke-Elder, 1997). O olho amétrope requer lentes divergentes, no caso da miopia, ou convergentes, no caso da hipermetropia, para que o foco do objeto atinja a retina (Netto, 2000). O astigmatismo ocorre quando diferentes regiões do sistema óptico falham em focalizar raios paralelos de luz de uma maneira uniforme. Isto pode ser devido às irregularidades regionais na curvatura da córnea ou do cristalino, que permitem que o raio de luz em um meridiano seja focalizado diferentemente do raio de luz de outro meridiano, resultando na deformação da imagem (Duke-Elder, 1997). O astigmatismo é considerado raro em cães, no entanto tem sido observado em uma variedade de raças (Prado, 1983; Miller e Murphy, 1995).

A afacia, após a facectomia, cria uma mudança significativa do estado de refração do olho (Pollet, 1982). Na oftalmologia humana, esse erro refracional pode ser corrigido pelo uso de óculos, lente de contato corneana ou, mais adequadamente, por lente intra-ocular (LIO) (Pollet, 1982). Na veterinária, são utilizadas lentes intra-oculares (LIOs), num esforço de maximizar a recuperação visual, com restauração da emetropia (Miller e Murphy, 1995). A implantação de duas ou mais lentes intraoculares na câmara posterior, polipseudofaquia ou em piggyback, é realizada para fornecer adequada correção óptica a pacientes pseudofácicos que requeiram alto poder de refração de lente intra-ocular, bem como para alcançar emetropia em olhos extremamente curtos (Findl et al., 1999; Gayton et al., 2000).

A prática padrão para pessoas submetidas à facectomia com implante de LIO é estimar o poder de dioptria, pelo uso de fórmulas matemáticas, por meio de ultrassonografia modo A e ceratometria; para tanto, cada equação contém uma variável adicional relatada para o tipo de lente intra-ocular e posição dentro do olho. Fórmulas de terceira geração, mais recentes, tais como Holladay, SRK-T e Hoffer Q, podem reduzir o erro refrativo pós-operatório (Donoso e Rodriguez, 2001).

A retinoscopia com luz em faixa, método prático e exato para o exame de refração (Netto, 2000), mostra-se inestimável em situações especiais, como crianças, pacientes surdos, senis, com transtornos mentais (Corboy, 1987), sendo de grande valia também em animais. Esse exame tem sido usado para definir o estado refrativo normal, patológico e induzido cirurgicamente nos olhos de cães, animais de laboratório, animais exóticos e outras espécies domésticas (Davidson, 1997). O método consiste em observar o movimento da faixa, refletido no fundo do olho, através da pupila; esses movimentos são neutralizados por lentes positivas ou negativas colocadas à frente do olho examinado (Corboy, 1987; Netto, 2000).

O presente trabalho teve por objetivo avaliar a eficácia da retinoscopia com luz em faixa para o uso em cães, assim como o erro refracional de animais fácicos, afácicos e pseudofácicos com implante de lente intra-ocular dupla (piggyback) e única (41D), determinando emetropia ou ametropia.

\section{MATERIAL E MÉTODO}

Empregaram-se 40 cães, machos e fêmeas, não diabéticos, divididos em quatro grupos de 10 cães cada, designados por cães fácicos $(\mathrm{CF})$, cães afácicos (CA), cães pseudofácicos com implante de duas lentes intra-oculares em piggyback (PP) e cães pseudofácicos com implante de uma lente intra-ocular veterinária de 41 D (PL); após aprovação pela Câmara Ética em Experimentação Animal da Faculdade de Medicina Veterinária e Zootecnia - Unesp Botucatu, SP.

As avaliações feitas no grupo $\mathrm{CF}$ e nos grupos CA, PP e PL, antes do procedimento cirúrgico, foram consideradas o primeiro momento do estudo (m0). Os demais momentos foram determinados com intervalos de 28 dias após a cirurgia (m28) e 60 dias após a cirurgia (m60). Após exame clínico geral e exame oftálmico completo, os cães que apresentaram enfermidades sistêmicas ou oculares, contraindicando a facectomia, foram retirados do experimento. 
Para realização da facectomia, a medicação préanestésica foi realizada com acepromazina $(0,05 \mathrm{mg} / \mathrm{kg}, \mathrm{IM})$ associada à morfina $(0,5 \mathrm{mg} / \mathrm{kg}$, IM), seguida de indução anestésica com propofol (5-7 mg/kg, IV) e manutenção com isoflurano. Foi instituída a ventilação com pressão positiva intermitente, após a administração de atracúrio $(0,05 \mathrm{mg} / \mathrm{kg}, \mathrm{IV})$. O bloqueio neuromuscular foi revertido com neostigmine $(0,07 \mathrm{mg} / \mathrm{kg}$, IV) e atropina $(0,04 \mathrm{mg} / \mathrm{kg}, \mathrm{IV})$.

A técnica cirúrgica adotada para remoção da catarata nos cães dos grupos CA, PP e PL foi a de facoemulsificação bimanual, feita por um cirurgião destro. Os do grupo $\mathrm{PP}$ receberam o implante de duas lentes de silicone dobrável intra-ocular ${ }^{1}$ em piggyback; nos animais do grupo PL, foi implantada uma única lente veterinária, acrílica hidrofílica de $41 \mathrm{D}^{2}$. Nesses, imediatamente ao término da cirurgia, foi aplicado $0,3 \mathrm{~mL}$ de ativador de plasminogênio tecidual (TPA) para dissolver a fibrina presente. $\mathrm{O}$ ato cirúrgico foi procedido unilateralmente.

$\mathrm{O}$ astigmatismo cirurgicamente induzido foi avaliado pelo autoceratômetro ${ }^{3}$, por meio de uma comparação entre três aferições consecutivas, nos eixos horizontal e vertical, em ambos os olhos, nos cães dos grupos CA, PP e PL. Os animais foram contidos manualmente, em estação ou sentados. $\mathrm{O}$ astigmatismo ceratométrico cirurgicamente induzido, nos mesmos grupos, foi calculado por análise vetorial pelo método de Cravy (Holladay et al., 1992) e suas médias, estatisticamente analisadas. O cálculo foi feito considerando-se as medidas ceratométricas no $\mathrm{m} 0$ e m60.

As mensurações oculares, com o animal sedado por levomepromazina $(0,5 \mathrm{mg} / \mathrm{kg} \quad \mathrm{IM}) \quad \mathrm{e}$ butorfanol $(0,2 \mathrm{mg} / \mathrm{kg} \mathrm{IM})$, foram realizadas por ultrassonografia modo-A, por meio de ecobiômetro ${ }^{4}$, com uma probe de $10 \mathrm{MHz}$. O aparelho foi calibrado com velocidades acústicas de $1526 \mathrm{~m} / \mathrm{s}$ para os humores aquoso e vítreo e $1710 \mathrm{~m} / \mathrm{s}$ para a lente, como proposto por Schiffer (1982). Realizou-se anestesia tópica corneana mediante instilação de cloridrato de

\footnotetext{
${ }^{1}$ Lente de silicone dobrável, SI40NB, PhacoFlex, Allergan, Brasil.

${ }^{2}$ Lente acrílica hidrofílica dobrável, C DOG SE, 41 dioptrias, Corneal do Brasil, Rio de Janeiro, Brasil.

${ }^{3}$ KM-500, Nidek Co., Japão.

${ }^{4}$ Echoscan US - 800, Nidek Co. - Japão.
}

proximetacaína. O transdutor foi posicionado em contato direto com o centro da córnea, e as mensurações oculares foram obtidas pela média de seis aferições consecutivas. Fez-se uma única avaliação em todos os grupos. Optou-se pela sedação, com contenção mecânica, associada à anestesia tópica para a realização da ecobiometria, para menor movimentação do bulbo ocular e melhor captação das imagens.

Para cálculo das lentes em piggyback, foi utilizada a fórmula indicada por Rodrigues (2004): $1^{\mathrm{a}} \mathrm{LIO}=60 \%$ do poder total da LIO, calculado pelo ecobiômetro pela fórmula SRK-T, utilizando a constante humana para lente de silicone de 118,0, considerando o poder desejado de $-1,0 \mathrm{D} ; 2^{\mathrm{a}} \mathrm{LIO}=40 \%$ do poder total da LIO, calculado pelo ecobiômetro, mais três dioptrias.

Para avaliação do erro refracional, foi realizada retinoscopia com luz em faixa, por um único examinador, em ambiente de baixa luminosidade, utilizando retinoscópio halógeno de faixa ${ }^{5}$ e réguas de esquiascopia ${ }^{6}$. Nos cães que não apresentaram midríase, mesmo após permanência em sala escura, foi feita dilatação pupilar aplicando-se colírio de cloridrato de tropicamida 1\%. Para realização da retinoscopia nos cães do grupo CA, foi necessária a confecção de uma lente asférica de $+14,75 \mathrm{D}$. A lente confeccionada foi posicionada por um auxiliar, bem próximo ao olho do animal (cerca de $1,2 \mathrm{~cm})$, e ao seu poder dióptrico foi adicionado o poder encontrado pela régua de retinoscopia. Para a realização da retinoscopia, foi fixado o manguito do retinoscópio em sua posição mais baixa (efeito de espelho plano), colocado a $66 \mathrm{~cm}$ do paciente. Nesta distância, foram usadas lentes de trabalho de $+1.5 \mathrm{D}$. Durante a retinoscopia, foram observados os movimentos do reflexo do fundo do olho e a direção do movimento - se na mesma direção da faixa (a favor) ou na direção oposta (contra). No movimento a favor, foram adicionadas lentes positivas, e no contra, acrescentaram-se lentes negativas até se obter $\mathrm{o}$ ponto de neutralização, quando a pupila se encheu de luz, sem nenhum movimento. A lente corretora que produzia o reflexo de neutralização era a que corrigia o erro de refração.

\footnotetext{
${ }^{5}$ Retinoscópio halógeno de faixa ${ }^{\circ} 18200$ de $3,5 \mathrm{v}$ - Welch Allyn, Ontário, Canadá.

${ }^{6}$ Paire de Règles à Skiascopie L2 - Convex et Concave.

Luneau Ophtalmologie - Chartres, França.
} 
Para a análise estatística, foram aplicados o teste Kruskall-Wallis (Tab. 1 e 2), análise de variância, seguida do teste Tukey para estudo do comprimento axial dos bulbos oculares, o teste $\mathrm{t}$ de Wilcoxon para estudo da hipermetropia e o coeficiente de correlação de Pearson para estudo das correlações. $\mathrm{O}$ nível de significância utilizado foi de $5 \%$.

Tabela 1. Mediana, primeiro e terceiro quartil referentes ao astigmatismo ceratométrico cirurgicamente induzido (em dioptrias cilíndricas negativas) obtido pela ceratometria, segundo os grupos

\begin{tabular}{ccccc}
\hline Variável & CA & PP & PL & Valor de P \\
\hline $\begin{array}{c}\text { Astigmatismo } \\
\text { induzido }\end{array}$ & $-0,79$ & $-1,30$ & $-0,85$ & 0,122 \\
\hline
\end{tabular}

CA: cães afácicos; PP: cães pseudofácicos com implante de duas lentes intra-oculares em piggyback; PL: cães pseudofácicos com implante de uma lente intra-ocular veterinária de 41D. Teste Kruskal-Wallis.

\section{RESULTADOS E DISCUSSÃO}

O astigmatismo corneano médio obtido pela ceratometria foi de $-0,42 \pm 0,20 \mathrm{D}$ no olho direito e $-0,47 \pm 0,36 \mathrm{D}$ no olho esquerdo dos animais do grupo $\mathrm{CF}(\mathrm{P}=0,74)$. Estes valores diferem dos de Soares (2002), que encontrou valor médio de astigmatismo de 1,02 $\pm 0,53$ em cães da raça Fila Brasileiro. Os resultados confirmam os obtidos por Miller e Murphy (1995), em que se verificou astigmatismo, variando de 0.5 a 3.0D, em 10 dos 240 cães estudados.

A média da curvatura corneana antes da cirurgia em todos os cães foi de 40,46 $\pm 2,44 \mathrm{D}$. Gaiddon et al. (1991) relataram que cães de grande porte exibem curvatura corneana menos acentuada $(37,35 \pm 1,67 \mathrm{D})$, quando comparados àqueles de médio $(40,62 \pm 1,44 \mathrm{D})$ e pequeno porte $(41,67 \pm 2,45 \mathrm{D})$. Considerando-se que a maioria dos cães usados era de pequeno porte, os resultados se assemelham aos encontrados por esses autores.

O comprimento axial dos bulbos oculares, utilizado para cálculo da fórmula SRK-T, foi diferente entre os grupos $(\mathrm{P}=0,01$; teste Tukey), sendo de $20,2 \pm 0,7 \mathrm{~mm}$ nos cães do grupo $\mathrm{CF}$, $20,6 \pm 1,2 \mathrm{~mm}$ nos do grupo CA, 19,5 $\pm 1,1 \mathrm{~mm}$ nos do grupo PP e $21,3 \pm 1,3 \mathrm{~mm}$ nos do grupo PL. Como essas avaliações foram realizadas antes da cirurgia, o fato de os animais pertencerem a qualquer dos grupos nada interfere no resultado do comprimento axial, visto que o tamanho relativo das estruturas intra-oculares do olho do cão é, parcialmente, geneticamente dependente (Ekesten e Torrang, 1995). Apesar de haver diferença entre os grupos, os valores da média do comprimento axial assemelham-se aos encontrados na literatura (Gaiddon et al., 1991; Murphy et al., 1992), estando dentro da normalidade.

Os cães do grupo CF apresentaram, em média, baixo grau de hipermetropia nos dois olhos, sendo de $0,25[-0,62 ; 1,37] \mathrm{D}$ no olho direito e de $0,56[-0,50 ; 1,50] \mathrm{D}$ no olho esquerdo $(\mathrm{P}=0,77$; teste Wilcoxon). Estes resultados assemelham-se aos obtidos por Murphy et al. (1992), Miller e Murphy (1995), Davidson (1997) e Soares (2002), nos quais foram observados leve hipermetropia em cães fácicos. No entanto, diferem dos de Pollet (1982), que observou ampla maioria de cães emétropes e dos de Murphy et al. (1992), Davidson (1997) e Mutti et al. (1999), que relataram miopia.

Nos cães do grupo CA, foi observado alto grau de hipermetropia, com o equivalente esférico de $19,87[17,00 ; 20,25] \mathrm{D}$ no $\mathrm{m} 28$ e $18,50[18,00$; 19,31]D no m60, mas não ocorreu diferença entre os momentos $(\mathrm{P}=0,164$; teste Wilcoxon). Estes resultados diferem dos encontrados por Pollet (1982), que relatou um erro de refração de 14D em olhos afácicos, mas aproximam-se do de Davidson et al. (1993), em um estudo feito com 48 olhos afácicos, cujo valor foi de $+14,14 \pm 2,10 \mathrm{D}$, com variação de $+10,5 \mathrm{a}+18,5 \mathrm{D}$.

Nos cães submetidos à facectomia, houve diferença entre grupos, quanto ao equivalente esférico no m60 (Tab. 2). Os animais do grupo $\mathrm{CA}$ diferiram dos animais do grupo $\mathrm{PL}$ $(\mathrm{P}=0,001)$. Nos do grupo $\mathrm{PP}$, ocorreram valores intermediários, não diferindo dos outros grupos CA e PL. 
Tabela 2. Mediana, primeiro e terceiro quartil referentes ao equivalente esférico (em dioptrias) obtido pela retinoscopia, segundo os grupos no $\mathrm{m} 60$

\begin{tabular}{ccccc}
\hline Variável & CA & PP & PL & Valor de P \\
\hline Equivalente & 18,50 & 5,25 & 2,00 & 0,001 \\
esférico & {$[18,00 ; 19,31] \mathrm{A}$} & {$[3,00 ; 6,50] \mathrm{AB}$} & {$[-0,81 ; 3,31] \mathrm{B}$} & \\
\hline
\end{tabular}

CA: cães afácicos; PP: cães pseudofácicos com implante de duas lentes intra-oculares em piggyback; PL: cães pseudofácicos com implante de uma lente intra-ocular veterinária de 41D. Teste Kruskal-Wallis.

Por ecobiometria, foi demonstrado que $37,8 \pm 4,0 \mathrm{D}$ foi o poder dióptrico necessário da LIO pela fórmula SRK-T com constante humana, para obter emetropia no grupo PP. Nesses animais foram encontrados valores de poder dióptrico na retinoscopia de 5,25 [3,00; 6,50]D. Nos cães do grupo PL, foi implantada uma LIO veterinária de $41 \mathrm{D}$ esse grupo apresentou baixo grau de hipermetropia residual, com 1,68[-0,50; $3,50] \mathrm{D}$ no $\mathrm{m} 28$ e $2,00[-0,81 ; 3,31] \mathrm{D}$ no $\mathrm{m} 60$, variando de $-0,81$ a $+3,50 \mathrm{D}$. Pode-se dizer que a lente intra-ocular utilizada que mais se aproximou da emetropia foi a de 41D, implantada nos cães do grupo PL, ao invés das duas lentes em piggyback, implantadas com auxílio da fórmula SRK-T com constante humana no grupo PP. Esses resultados sugerem estudos no sentido da aplicação de outra fórmula para o cálculo de lentes intra-oculares em cães. A fórmula SRK-T é indicada para olhos com comprimento axial curto, encontrado em cães, como o citado por Donoso e Rodriguez (2001). No entanto, novas fórmulas, como a de Holladay II, irão provavelmente possibilitar o cálculo da LIO, mais precisamente nesses casos, por considerarem outros parâmetros, como a profundidade da câmara anterior, a espessura do cristalino e o diâmetro corneano (Donoso e Rodriguez, 2001). A literatura a esse respeito propõe que o mais adequado é calcular o poder dióptrico de LIO individualmente. Se isso não for possível, recomenda-se o uso de uma LIO próxima de 41,5D (Gaiddon et al., 1991; Davidson et al., 1993; Gaiddon et al., 1997).

É antigo o consenso que quanto menor o tamanho da incisão menor o astigmatismo após a cirurgia e, portanto, melhor a acuidade visual (Oshika et al., 1994). O astigmatismo ceratométrico cirurgicamente induzido foi baixo (Tab. 1), o que pode ter sido ocasionado pelo tamanho da incisão corneana $(3,2 \mathrm{~mm})$, que não diferiu entre os grupos com e sem implante de lente intra-ocular, bem como pela experiência do cirurgião em manter correto posicionamento das mãos, evitando o estresse cirúrgico na incisão.
Os resultados diferem dos de Nelms et al. (1994), pois foram encontrados valores mais baixos que os citados com acesso corneano, aos 60 dias de pós-operatório, demonstrando que o acesso em córnea clara induziu pouco astigmatismo. O menor astigmatismo induzido foi o do grupo CA, enquanto o maior foi o do grupo PP, resultante provavelmente das complicações observadas nos referidos grupos.

Segundo Duke-Elder (1997) e Soares (2002), o ceratômetro não é um método absoluto de avaliação do astigmatismo corneano durante a refração. Recomenda-se que o instrumento seja considerado um método de estimativa do grau corneano, que pode ser conseguido de forma rápida, em mãos experientes. A leitura não dá o valor do astigmatismo total necessário para as lentes corretoras, porém o do cilindro que, quando colocado em contato com a córnea, corrige a curvatura astigmática da sua superfície. Partindo desse pressuposto, o astigmatismo encontrado, por ceratometria, pode ser diferente do encontrado pela retinoscopia, devido à falta de precisão do primeiro exame, omitindo-se superfícies refratoras do bulbo ocular. Nos animais do grupo $\mathrm{CF}$, não se observou correlação entre o astigmatismo da ceratometria e o da retinoscopia nos olhos direito e esquerdo ( $\mathrm{r}=0,22$; coeficiente de correlação de Pearson, $\mathrm{P}=0,54)$. Nos dos grupos CA, PP e PL, a correlação foi significativa, porém baixa $(\mathrm{r}=0,30$; coeficiente de correlação de Pearson, $\mathrm{P}=0,035$ ).

$\mathrm{Na}$ retinoscopia com luz em faixa, a contenção mecânica mostrou-se suficiente para a realização dos exames. $\mathrm{O}$ uso do retinoscópio nas opacificações do meio ou nas irregularidades da córnea fica impossibilitado (Corboy, 1987) e a realização da retinoscopia pode ser muito dificultada em pacientes afácicos e pseudofácicos, com opacidades causadas por edema corneano, flare aquoso e opacificação capsular (Davidson, 1997), variabilidade no tamanho pupilar, reflexos vindos do implante e 
fibrose e enrugamento na cápsula (Peiffer e Gaiddon, 1991).

Neste estudo, algumas complicações causadas pela facectomia dificultaram e, muitas vezes, impediram a realização da retinoscopia. Nos cães pseudofácicos, a reação inflamatória foi bem mais intensa se comparados aos afácicos. Nos cães do grupo PP, as complicações foram piores do que nos do grupo PL. Como ambos os grupos tiveram técnica e cirurgião padronizados, é possível considerar o fato de que a implantação de duas lentes em piggyback determine uma reação inflamatória maior. Em todos os animais desse grupo, não foi possível a realização da retinoscopia aos 28 dias, pela impossibilidade de visualização do fundo ocular com nitidez. Foram observadas presença de traves de fibrina na câmara anterior, sobre as LIOs, opacidade de cápsula anterior, sinéquias, úlceras de córnea, floater vítreo e, em casos mais severos, alteração corneana determinando diminuição de sua transparência, iris bombé, e hipertensão com glaucoma secundário. Em animais sem vitrectomia anterior, a uveíte também foi menor, facilitando a realização da retinoscopia. Tendo em vista que nos cães do grupo $\mathrm{PP}$ não foi possível a retinoscopia em vários momentos, nos do grupo PL optou-se por medidas auxiliares durante a cirurgia, para viabilizar a retinoscopia aos 28 e 60 dias, como a aplicação de TPA, que dissolveu a fibrina na câmara anterior.

A cicloplegia é rotineiramente usada para relaxar a acomodação, quando se faz a refração em crianças. Como a maioria dos animais domésticos tem habilidade de acomodação limitada, a cicloplegia é geralmente desnecessária (Murphy et al., 1992; Miller e Murphy, 1995; Davidson, 1997). Valores de retinoscopia com ou sem cicloplegia foram similares em um estudo de refração conduzido em cães (Davidson, 1997).

Segundo Davidson (1997), na oftalmologia veterinária e pediátrica, a retinoscopia necessita da cooperação do paciente e da destreza do examinador, o que torna o exame desafiador em virtude da constante movimentação do paciente. Este foi um aspecto crítico durante os exames, o que deve ser levado em consideração antes da escolha do paciente que será submetido à retinoscopia. Pacientes agitados e com alterações neurológicas dificultam demasiadamente a realização do exame.

Uma possibilidade para melhorar a confiabilidade da retinoscopia é aumentar o número de repetições e o número de examinadores. A experiência do examinador e a cooperação do paciente são críticas para resultados mais confiáveis. Um erro de refração de $\pm 0,5 \mathrm{D} \quad$ mostra-se aceitável entre oftalmologistas veterinários e pode ser obtido com moderado grau de experiência do examinador. Cuidados na neutralidade, alinhamento óptico e manutenção de uma distância correta entre o examinador e o paciente são fatores importantes para alcançar a refração (Davidson, 1997).

\section{CONCLUSÕES}

A retinoscopia com luz em faixa é um método eficaz para avaliar a refração ocular em cães, mas é dificultada quando na presença de opacidade de meios oculares. Cães fácicos apresentam menor erro refracional, com discreto grau de hipermetropia, e cães afácicos apresentam alto grau de hipermetropia. Cães com implante de lente dupla, em piggyback, calculada pela fórmula SRK-T, apresentam moderado grau de hipermetropia, e cães com implante de lente única com 41D apresentam erro refracional mais próximo ao obtido em cães fácicos. $\mathrm{O}$ astigmatismo ceratométrico cirurgicamente induzido após facoemulsificação em córnea clara é discreto, e a fórmula SRK-T, utilizada com constante humana, não é adequada para o cálculo biométrico em cães, pois induziu alto erro hipermetrópico.

\section{AGRADECIMENTO}

À Fundação de Amparo à Pesquisa do Estado de São Paulo (FAPESP), pelo apoio financeiro $\left(\mathrm{n}^{\circ}\right.$ 2004/00620-0).

\section{REFERÊNCIAS BIBLIOGRÁFICAS}

CORBOY, J.M. Manual prático da retinoscopia. Rio de Janeiro: Colina, 1987. 199p.

DAVIDSON, M.G. Clinical retinoscopy for the veterinary ophthalmologist. Vet. Comp. Ophthalmol., v.7, p.128-137, 1997. 
DAVIDSON, M.G.; MURPHY, C.J.; NASISSE, M.P. et al. Refractive state of aphakic and pseudophakic eyes of dogs. Am. J. Vet. Res., v.54, p.174-177, 1993.

DONOSO, R.; RODRIGUEZ, A. Piggyback implantation using the AMO Array multifocal intraocular lens. J. Cataract Refract. Surg., v.27, p.1506-1510, 2001.

DUKE-ELDER, S. Refração prática. Rio de Janeiro: Rio Med Livros, 1997. 306p.

EKESTEN, B.; TORRANG, I. Heritability of the depth of the opening of the ciliary cleft in Samoyeds. Am. J. Vet. Res., v.56, p.1138-1143, 1995.

FINDL, O.; MENPACE, R.; RAINER, G. et al. Contact zone of piggyback acrylic intraocular lenses. J. Cataract. Refract. Surg., v.25, p.860$862,1999$.

GAIDDON, J.; ROSOLEN, S.G.; STERU, L. et al. Use of biometry and keratometry for determining optimal power for intraocular lens implants in dogs. Am. J. Vet. Res., v.52, p.781783, 1991.

GAIDDON, J.; LALLEMENT, P.E.; BOUHANA, N. Study of refraction by retinoscopy of normal, aphakic and pseudophakic canine eyes: advantages of using a 41 diopter intraocular lens. Prat. Med. Chir. Anim. Comp., v.32, p.531-535, 1997.

GAYTON, J.L.; APPLE, D.J.; PEN, Q. et al. Interlenticular opacification: clinicopathological correlation of a complication of posterior chamber piggyback intraocular lenses. $J$. Cataract. Refract. Surg., v.26, p.330-336, 2000.

GELATT, K.N. Veterinary Ophthalmology. 3.ed. Philadelphia: Lippincott Williams \& Wilkins, 1999. 1544p.

HOLLADAY, J.T.; CRAVY, T.V.; KOCH, D.D. Calculating the surgically induced refractive change following ocular surgery. J. Cataract. Refract. Surg., v.18, 1992, p.429-443.

MILLER, P.E.; MURPHY, C.J. Vision in dogs. J. Am. Vet. Med. Assoc., v.207, p.1623-1633, 1995.

MURPHY, C.J.; ZADNIK, K.; MANNIS, M.J. Myopia and refractive error in dogs. Invest. Ophthalmol. Visual Sci., v.33, p.2459-2463, 1992.
MURPHY, C.J.; MUTTI, D.O.; ZADNIK, K. et al. Effect of optical defocus on visual acuity in dogs. Am. J. Vet. Res., v.58, p.414-418, 1997.

MUTTI, D.O.; ZADNIK, K.; MURPHY, C.J. Naturally occurring vitreous chamber based myopia in Labrador retriever. Invest. Ophthalmol. Visual Sci., v.40, p.1577-1584, 1999.

NELMS, S.R.; DAVIDSON, M.G.; NASISSE, M.P. et al. Comparison of corneal and escleral surgical approaches for cataract extraction by phacoemulsification and intraocular lens implantation in normal dogs. Vet. Comp. Ophthalmol., v.4, p.53-60, 1994.

NETTO, A.L. Emetropias e ametropias: testes objetivos e subjetivos. In: URAS, R.O. Óptica e refração ocular. Rio de Janeiro: Cultura Médica, 2000. p.51-65.

OSHIKA, T.; TSUBOI, S.; YAGUCHI, S. et al. Comparative study of intraocular lens implantation through 3,2 and 5,5 mm incisions. Ophthalmology, v.101, p.1183-1190, 1994.

PEIFFER, R.L.; GAIDDON, J. Posterior chamber intraocular lens implantation in the dog: results of 65 implants in 61 patients. J. Am. Anim. Hosp. Assoc., v.27, p.453-462, 1991.

POLLET, L. Refraction of normal and aphakic canine eyes. J. Am. Anim. Hosp. Assoc., v.18, p.323-326, 1982.

PRADO, D. Noções de óptica, refração ocular e adaptação de óculos. São Paulo: Ótica Revista, 1983. v.2, p.19-110.

RODRIGUES, G.N. Estudo clínico da facoemulsificação em cães, com e sem implante de lente intra-ocular em piggyback. 2004. 175 f. Tese (Doutorado) - Faculdade de Medicina Veterinária e Zootecnia, Universidade Estadual Paulista, Botucatu.

SCHIFFER, S.P. Biometry study of the canine eye, using a-mode ultrasonography. Am. J. Vet. Res., v.43, p.23-26, 1982.

SOARES, A.M.B. Ceratometria, retinoscopia e ecobiometria do bulbo do olho de cães da raça Fila Brasileiro. 2002. 60f. Tese (Doutorado) Faculdade de Ciências Agrárias e Veterinárias, Universidade Estadual Paulista, Jaboticabal. 